\title{
Seroprevalence of porcine cysticercosis and influence of some associated risk factors in Northwestern Romania
}

\author{
Ana-Maria Oleleu ${ }^{1,2}$, Călin Mircea Gherman ${ }^{1}$, Radu Blaga $^{3}$, Adriana Györke $^{1}$, \\ Vasile Cozma ${ }^{1,4}$ \\ ${ }^{1}$ University of Agricultural Sciences and Veterinary Medicine Cluj-Napoca, Faculty of Veterinary Medicine, \\ Cluj-Napoca, Cluj County, Romania \\ ${ }^{2}$ Sanitary Veterinary And Food Safety Agency (SVFSD), Cluj-Napoca, Cluj, Romania \\ ${ }^{3}$ Alfort Veterinary School, Maisons Alfort Cedex, France \\ ${ }^{4}$ Academy of Agricultural and Forestry Sciences, Bucharest, Romania \\ Received August 13, 2015 \\ Accepted June 1, 2016
}

\begin{abstract}
Cysticercosis is a zoonotic disease that may have a significant impact on the public health. The aim of the current study was to assess the seroprevalence of porcine cysticercosis in Northwestern Romania and the influence of several risk factors such as age, sex and season. The study included 1,288 pigs originating from 7 different counties and was conducted between May 2014 and May 2015. All pigs were reared in the traditional backyard pig system. Of these, 226 were young and 1,062 were adult pigs, 188 were males and 1,100 females. Serum samples were collected. The apDia Cysticercosis Antigen ELISA (apDia, Belgium) commercial kit was used to identify the viable cysticerci of Taenia sp. Out of the 1,288 serum samples, 83 were seropositive (seroprevalence: $6.4 \%)$. The risk factors' analysis showed that age $(3.1 \%$ juveniles vs $7.1 \%$ adults $[P<0.03])$ and season $(9.3 \%$ fall vs $11.8 \%$ winter $[P<0.00001] ; 3.4 \%$ spring vs $1.2 \%$ summer $[P<0.00001])$ had a significant influence on the seropositivity, while sex had no significant influence $(3.7 \%$ males vs $6.9 \%$ females, $P=0.1)$. Considering the genus specificity of ELISA commercial kit, the serology should be correlated with the post mortem examination in order to confirm the disease and have a Cysticercus cellulosae specific prevalence.
\end{abstract}

Pigs, ELISA, prevalence, age, sex, season

Cysticercosis is a tissue infection caused by the larval stage of an adult tapeworm. In pigs, two different diseases have been described, the visceral cysticercosis caused by the metacestode stage of Taenia hydatigena (previously known as Cysticercus tenuicollis, definitive hosts: canids) and the muscular cysticercosis produced by the metacestode stage of Taenia solium (formerly known as Cysticercus cellulosae, definitive hosts: humans) (Tsang and Wilson 1995). Accidentally, humans become an aberrant intermediate host of $C$. cellulosae and develop subsequent neurocysticercosis because of the faecal-oral transmission through person-to-person contact, autoinfection or consumption of contaminated food. This neglected, emerging or re-emerging disease is mostly diagnosed in developing countries (Cantey et al. 2014). Meat processing industry and pork meat consumption are economically affected by these metacestodes (Pawlowski et al. 2005).

Gaps in knowledge and control of these diseases are still present despite the emerging or re-emerging character of the muscle infection in different regions of the world. Although the $T$. solium/metacestode stage of $T$. solium complex in humans and pigs is important, neurocysticercosis is rarely reported in Romania (Dulamea 2015). Moreover, the backyard system of rearing pigs, known as a major risk factor in the epidemiology of porcine cysticercosis, is still prevalent in Romania. 
The purpose of this study was to evaluate the seroprevalence of cysticercosis in pigs bred in the traditional pig farming system in Northwest Romania and to assess the influence of some risk factors such as the season, age and sex.

\section{Materials and Methods}

Study area and time period

The study was conducted in pigs bred in the traditional outdoor system in seven counties of Northwestern Romania, between May 2014 and May 2015. The animals had access to pasture. Four villages were randomly selected in each county for sampling: Bihor (BH), Bistrița-Năsăud (BN), Cluj (CJ), Maramureș (MM), Mureș (MS), Sălaj (SJ) and Satu-Mare (SM) (Fig. 1).

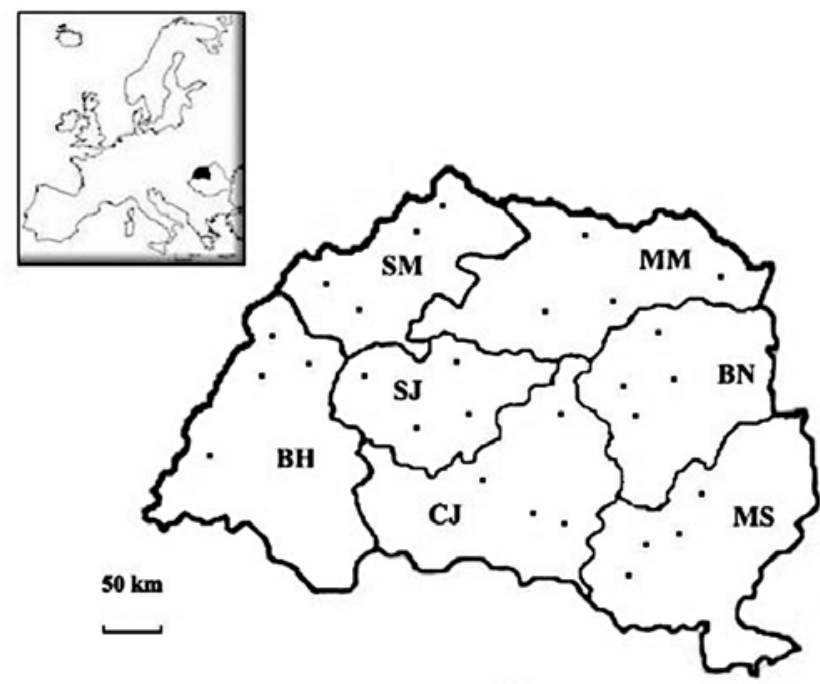

Fig. 1. The geographical origin of the collected samples from Northwestern Romania ( $\cdot$ collection sites)

\section{Collecting and processing of the samples}

Blood samples were collected from the jugular vein. The serum was separated from the clot to avoid haemolysis and preserved by freezing at $-20^{\circ} \mathrm{C}$ until use.

The apDiaCysticercosis Antigen (Ag) ELISA (apDia, Belgium), a genospecific test, was used to detect viable cysticerci of Taenia sp. from the serum samples. The kit has monoclonal antibodies (IgG isotype) against excretory-secretory products (ESP) of viable T. saginata cysticerci (Brandt et al. 1992; Dorny et al. 2004). Each sample, including positive and negative control, was double tested according to the manufacturer's instructions.

The test is considered valid if the individual absorbance value of the optical density (OD) is $>1.000$ for the positive control and $<0.100$ for the negative control. According to the kit specifications, serum samples with $\mathrm{Ag}$ Index $\leq 0.8$ were considered negative, $\geq 1.3$ positive, and an Ag Index varying between 0.8 and 1.3 was considered doubtful.

\section{Statistical analysis}

The prevalence and frequency distribution of the overall infection were tested by Epi Info software using Chi-square test. The analysis was performed considering independent variables such as age $(\leq 12$ months old, $>12$ months old), sex (males and females), and season (fall, winter, spring, and summer). $P$ was considered significant if $<0.05$.

\section{Ethics}

Serum samples were collected within The Surveillance, Prevention, Control and Eradication of Animal Diseases National Program; therefore, the approval from the Ethics Commission of the University of Agricultural Sciences and Veterinary Medicine, Cluj-Napoca, Romania was not required. 


\section{Results}

\section{Collected samples}

A total number of 1,288 samples were collected from all counties during the 4 seasons. The age of animals ranged between 6 and 44 months. They were divided into 2 age categories: youth 6-12 months old and adults $>12$ months old. Of all samples, 226 were collected from young animals and 1,062 from adults, and 188 from males and 1,100 from females.

\section{Seroprevalence of porcine cysticercosis}

The ELISA test was validated by an OD of 2.305 for the positive control and 0.66 for the negative control. The values of positive samples ranged between 1.756 and 3.123 , and of negative samples between 0.063 and 0.126 . No doubtful results were recorded.

Out of the 1,288 serum samples tested, 83 were seropositive; the overall prevalence was $6.4 \%$. The Cysticercus sp. infection was diagnosed in all territorial units. The lowest prevalence was recorded in the Bistrița-Năsăud county $(3.3 \%, 95 \%$ CI: $1.2-7.0)$ and the highest in Cluj County (11.4\%, 95\% CI: 7.2-16.9) (Table 1).

Table 1. Seroprevalence of T. solium cysticercosis in pigs by Ag-ELISA examination in Northwestern Romania.

\begin{tabular}{|c|c|c|c|c|c|}
\hline Factor & & Frequency & Prevalence $(\%)$ & $95 \% \mathrm{CI}$ & $P$-value \\
\hline \multirow{7}{*}{ County } & $\mathrm{BH}(n=184)$ & 8 & 4.3 & $1.9-8.4$ & \multirow{7}{*}{$0.03^{* *}$} \\
\hline & $\mathrm{BN}(n=184)$ & 6 & 3.3 & $1.2-7.0$ & \\
\hline & $\mathrm{CJ}(n=184)$ & 21 & 11.4 & $7.2-16.9$ & \\
\hline & $\operatorname{MM}(n=184)$ & 12 & 6.5 & $3.4-11.1$ & \\
\hline & $\operatorname{MS}(n=184)$ & 10 & 5.4 & $2.6-9.8$ & \\
\hline & $\mathrm{SJ}(n=184)$ & 10 & 5.4 & $2.6-9.8$ & \\
\hline & $\mathrm{SM}(n=184)$ & 16 & 8.7 & $5.1-13.7$ & \\
\hline \multirow{4}{*}{ Season } & Fall $(n=322)$ & 30 & 9.3 & $6.5-13.2$ & \multirow[t]{4}{*}{$0.00001^{* * *}$} \\
\hline & Winter $(n=322)$ & 38 & 11.8 & $8.6-16.0$ & \\
\hline & Spring $(n=322)$ & 11 & 3.4 & $1.8-6.2$ & \\
\hline & Summer $(n=322)$ & 4 & 1.2 & $0.4-3.4$ & \\
\hline \multirow{2}{*}{ Age class } & Grower (6-12 months) $(n=226)$ & 7 & 3.1 & $1.3-6.3$ & \multirow[b]{2}{*}{$0.03 * *$} \\
\hline & Adult ( $\geq 12$ months) $(n=1,062)$ & 76 & 7.2 & $5.7-8.9$ & \\
\hline \multirow{2}{*}{ Sex } & Females $(n=1,100)$ & 76 & 6.9 & $5.5-8.6$ & \multirow[t]{2}{*}{$0.1^{*}$} \\
\hline & Males $(n=188)$ & 7 & 3.7 & $1.5-7.5$ & \\
\hline
\end{tabular}

$\mathrm{n}$ - number of specimens; CI - Confidence Interval; ${ }^{*}$ Non-significant; ${ }^{* *}$ Significant $(P<0.05)$; ${ }^{* * *}$ Significant $(P<0.01)$

Risk factors analysis in the study area

Seasonal seroprevalence of Cysticercus sp. infection was higher in fall (30/322 [9.3\%, 95\% CI: 6.5-13.21]) and winter (38/322 [11.8\%, 95\% CI: 8.6-16.0] compared to spring (11/322, [3.4\%, 95\% CI: 1.8-6.2]) and summer (4/322, [1.24\%, 95\% CI: $0.4-3.4])$ (Table 1). The differences between fall vs winter, and spring vs summer, respectively, were significant $(P<0.00001)$.

Based on the age, the prevalence of porcine cysticercosis was 7/226 (3.1\% [95\% CI: $1.3-$ $6.3])$ in the 6-12 months age category and 76/106 (7.2\% [95\% CI: 5.7-8.9]) in the > 12 months age category (Table 1). The difference between the age categories was significant $(P<0.03)$.

Based on sex, the seroprevalence was $7 / 188(3.72 \%, 95 \% \mathrm{CI}: 1.5-7.5)$ for males and 76/1100 (6.9\% [95\% CI: 5.5-8.6]) for females. The statistical analysis showed no significant difference (Table 1). 


\section{Discussion}

The $T$. solium taeniasis/cysticercosis complex is a prevalent disease worldwide, with a hyperendemic distribution in many developing countries in Africa and Asia, being rarely diagnosed in North America, Europe, and Australia (Rajshekhar et al. 2003; Bouteille 2014). In 2010, Dorny et al. reported porcine cysticercosis in five Europian countries: Austria, Estonia, Lithuania, Poland, and Romania. However, up to date, limited data are available regarding the visceral or subserosal cysticercosis prevalence in pigs. Different studies showed an $8.30 \%$ prevalence in Uttar Pradesh (India) (Pathak and Gaur 1982), 3.22\% in Jos Plateau (Nigeria) (Fabiyi 1979) and 50.8\% in New Zealand (Ines on 1953).

Given the potential impact of this disease on public health, our study is the first regional screening designed to assess seroprevalence of porcine cysticercosis in Romania. We identified an overall seroprevalence of $6.4 \%$. The apDia Cysticercosis Ag ELISA kit (apDia Belgium) we used has a specificity of $99.6 \%$ for pig serum samples (Cysticercosis AG Elisa Technical Guide 2014). Despite its high sensitivity, the assay is genus-specific and does not allow the differentiation between T. solium and T. hydatigena larvae infections in pigs. Therefore, we may also have positive crossed reactions between these two larvae. Even so, the seroprevalence identified in this study was lower than the serosal cysticercosis prevalence (12\%) reported in wild boar in Romania (Cucoranu 2010).

Emergence or re-emergence of Taenia sp. larvae infection in pigs is influenced by several risk factors, mainly by poor sanitation, the pig rearing system and their slaughter within the household under unsuitable conditions (Enander et al. 2010; Gabriël et al. 2015). In our study, the main risk factor is considered poor sanitation in the selected locations because of low health education; in some cases, animals even have access to human excrements. This may support the high prevalence found in the pigs in our study. However, the reported morbidity of taeniasis in Romania is low in humans (ranges: $0.4 \%$ and $0.75 \%$ ), but a high prevalence of Taenia sp. infections was reported in dogs $(1.78 \%$ in southern Romania, Costin et al. 2011). The latter may contribute to a significant extent to environmental pollution and contamination of pigs, but it would be interesting also to screen the dogs in the selected areas of our study.

Traditional backyard pig rearing with outdoor access is widely spread in Transylvania where the pigs are raised in coops made of solid material, generally concrete. Sanitation measures are easily applicable and consist of removing the faeces and washing the floor daily. Given this, the chance of contact between pigs and the parasites' eggs eliminated by humans or dogs is low. However, the practice of feeding pigs with green mass harvested from the pasture creates an opportunity for this contact. Pollution of these pastures with Taenia sp. eggs is possible as some persons defecate outside, in the environment, or by subsequent contamination from stray and shepherd dogs. In addition, the pigs from mountain hamlets are freely reared on these contaminated pastures, which increases the contamination risk.

Another common risk factor in Romania is the traditional home slaughter of pigs especially during the winter holidays, performed by the owners. Local legislative regulations only request meat examination by accredited veterinarians for trichinellosis, but the pillar muscles of the diaphragm are not the preferred sites of T. solium. The possibly infected viscera are usually given uncooked to dogs. This way, the biological cycle of cestodes is maintained in the area.

The influence of age as a risk factor in the prevalence of infection in pigs is supported by the animals' exposure throughout their lifetime to accidental consumption of tapeworm eggs. It has been shown that the prevalence slowly increases from $46.8 \%$ in piglets of 2-4 months to $80.9 \%$ in adults over 1 year in Peru (Garcia et al. 2003). We have found 
a similar significant increase with age: the prevalence in adults $(7.15 \%)$ was 2 -fold higher than in young pigs $(3.1 \%)$.

Regarding the sex, different studies suggest that this factor has no influence on cysticercosis prevalence in pigs (Ngwing et al. 2012; Yulianto et al. 2014). Our results were similar to previous findings. The difference between the seroprevalence in males and females $(3.7 \%$ versus $6.9 \%)$ was not significant.

Seasonal influence of cysticercosis prevalence in pigs may be explained by 1) higher frequency of human faeces consumption by pigs (Copado et al. 2004) and increased frequency of the farmers' defecation in the field in the dry season (Yulianto et al. 2014); 2) pigs housing in the warm, sunny and dry season, when they are left free to graze around households or garbage sites (Kagira et al. 2010). In our study, the higher seroprevalence was recorded during autumn fall $(9.3 \%)$ and winter $(11.8 \%)$. As the biology of larval forms requires 2 to 3 months to complete the development stages (Stewart and Hoyt 2006), it means that seropositive animals were contaminated during the spring and summer, when pigs graze on pasture.

This study has shown that porcine cysticercosis is present in Northwestern Romania and that age and season are significant risk factors in traditional backyard rearing system of pigs.

\section{Acknowledgements}

This paper was published within the European Social Fund, Human Resources Development Operational Programme 2007-2013, project no. POSDRU/159/1.5/136893.

\section{References}

Bouteille B 2014: Épidémiologie de la cysticercose et de la neurocysticercose. Med Sante Trop 24: 367-374

Brandt JR, Geerts S, De Deken R, Kumar V, Ceulemans F, Brijs L, Falla N 1992: A monoclonal antibodybased ELISA for the detection of circulating excretory-secretory antigens in Taenia saginata cysticercosis. Int J Parasitol 22: 471-477

Cantey PT, Coyle CM, Sorvillo FJ, Wilkins PP, Starr MC, Nash TE 2014: Neglected parasitic infections in the United States: cysticercosis. Am J Trop Med Hyg 90: 805-809

Copado F, Aluja de AS, Mayagoitia L, Galindo F 2004: The behaviour of free-ranging pigs in the Mexican tropics and its relationships with human faeces consumption. Appl Anim Behav Sci 88: 243-252

Costin II, Enachescu V, Ciopaşiu R, Ioniţă M, Mitrea IL 2011: Prevalence of endoparasites, by coproscopical examination, on dogs from some urban and rural areas in south of Romania. Sci Papers Vet Med Timişoara 44: $31-37$

Cucoranu D 2010: The study of gastrointestinal nematodes in pigs in Mures county. Ph.D. Thesis, Cluj-Napoca, $341 \mathrm{p}$.

Cysticercosis AG Elisa Technical Guide 2014: apDia, Raadsherenstraat 3 - 2300 Turnhout, Belgium

Dorny P, Phiri IK, Vercruysse J, Gabriel S, Willingham III AL, Brandt J, Victor B, Speybroeck N, Berkvens D 2004: A Bayesian approach for estimating values for prevalence and diagnostic test characteristics of porcine cysticercosis. Int J Parasitol 34: 569-576

Dorny P, Vallée I, Alban L, Boes J, Boireau P, Boué F, Claes M, Cook AJC, Enemark H, Giessen van der J, Hunt KR, Howell M, Kirjušina M, Nőckler K, Pozio E, Rossi P, Snow L, Taylor MA, Theodoropoulos G, VieiraPinto MM, Zimmer IA 2010: Scientific Report submitted to EFSA. Development of harmonised schemes for the monitoring and reporting of Cysticercus in animals and foodstuffs in the European Union. European Food Safety Authority. Parma.

Dulamea AO 2015: Neurocysticercosis presenting with paroxysmal sensory disturbance. Rom J Neurol 14: 98-100

Enander RT, Amaya AR, Enander RA, Gute DM 2010: Neurocysticercosis: risk and primary prevention strategies update. Int J Environ Health Res 20: 329-365

Fabiyi JP 1979: Helminths of the pig on the Jos Plateau, Nigeria: relative prevalence, abundance, and economic significance. J Helminthol 53: 65-71

Gabriël S, Johansen MV, Pozio E, Smit GS, Devleesschauwer B, Allepuz A, Papadopoulos E, van der Giessen J, Dorny P 2015: Human migration and pig/pork import in the European Union: What are the implications for Taenia solium infections? Vet Parasitol 213: 38-45

Garcia HH, Gilman RH, Gonzalez AE, Verastegui M, Rodriguez S, Gavidia C, Tsang VC, Falcon N, Lescano AG, Moulton LH, Bernal T, Tovar M, Cysticercosis Working Group in Perú 2003: Hyperendemic human and porcine Taenia solium infection in Perú. Am J Trop Med Hyg 68: 268-275 
Ineson MJ 1953: A Comparison of the parasites of wild and domestic pigs in New Zealand. Trans N Z Inst 82: 579-609

Kagira JM, Maingi N, Kanyari PW, Githigia SM, Ng'ang'a JC, Gachohi JM 2010: Seroprevalence of Cysticercus cellulosae and associated risk factors in free-range pigs in Kenya. J Helminthol 84: 398-403

Ngwing NA, Poné JW, Mbida M, Pagnah AZ, Njakoi H, Bilong CF 2012: A preliminary analysis of some epidemiological factors involved in porcine cysticercosis in Bafut and Santa subdivisions, North West Region of Cameroon. Asian Pac J Trop Med 5: 814-817

Pathak KM, Gaur SN 1982: The incidence of adult and larval stage Taenia hydatigena in Uttar Pradesh (India). Vet Parasitol 10: 91-95

Pawlowski Z, Allan J, Sart E 2005: Control of Taenia solium taeniasis/cysticercosis: From research towards implementation. Int J Parasitol 35: 1221-1232

Rajshekhar V, Joshi DD, Doanh NQ, van De N, Xiaonong Z 2003: Taenia solium taeniosis/cysticercosis in Asia: epidemiology, impact and issues. Acta Trop 87: 53-60

Stewart TB, Hoyt PG 2006: Internal Parasites. In: Straw BE, Zimmerman JJ, D’Allaire S, Taylor DJ (eds): Diseases of Swine, $9^{\text {th }}$ edn. Blackwell Publishing, Ames, pp. 901-915

Tsang VCW, Wilson M 1995: Taenia solium cysticercosis: an under-recognized but serious public health problem. Parasitol Today 11: 125-126

Yulianto HH, Satrija F, Lukman DW, Sudarwanto M 2014: Seroprevalence and risk factors of porcine cysticercosis in Way Kanan District, Lampung Province, Indonesia. Global Vet 12: 774-781 